\title{
Three Nematode Species Recovered from Terrestrial Snakes in Republic of Korea
}

\author{
Seongjun Choe', Junsik Lim², Hyun Kim², Youngjun Kim³ , Heejong Kim, Dongmin Lee', Hansol Park, \\ Hyeong-Kyu Jeon ${ }^{1}$, Keeseon S. Eom ${ }^{1, *}$ \\ 'Department of Parasitology, Medical Research Institute and Parasite Resource Bank, Chungbuk National University, School of Medicine, Cheongju \\ 28644, Korea; ' ${ }^{2}$ College of Veterinary Medicine, Kyungpook National University, Daegu 41566, Korea; ${ }^{3}$ National Institute of Ecology, Seocheon, \\ Chungnam 33657, Korea; ${ }^{4}$ Chungnam Wild Animal Rescue Center, Kongju National University, Yesan, Chungnam 32439, Korea
}

\begin{abstract}
The majority of parasitological studies of terrestrial snakes in Korea have focused on zoonotic parasites. However, in the present study, we describe 3 unrecorded nematode species recovered from 5 species of snakes $(n=6)$ in Korea. The examined snakes, all confiscated from illegal hunters, were donated by the Chungnam Wild Animal Rescue Center and Korean Broadcasting System in July 2014 and February 2015. Light and scanning electron microscopies on the shapes of spicules that are either bent or straight (kalicephalids) and the presence of the intestinal cecum (ophidascarids) figured out 3 nematodes; Kalicephalus brachycephalus Maplestone, 1931, Kalicephalus sinensis Hsü, 1934, and Ophidascaris excavata Hsü and Hoeppli, 1934. These 3 species of nematode faunas are recorded for the first time in Korea.
\end{abstract}

Key words: Kalicephalus brachycephalus, Kalicephalus sinensis, Ophidascaris excavata, nematode, snake, Korea

Terrestrial snakes are known to be the infection source of zoonotic parasites, such as sparganum, Neodiplostomum seoulense, Mesocestoides spp., Gnathostoma hispidum, and G. nipponicum, in the Republic of Korea (Korea) [1-6]. The majority of studies on the snake parasites were focused on zoonotic helminths with the public health concern.

As nematodes detected from the snake hosts, Kalicephalus natricis and 2 species of larval Gnathostoma, i.e., G. hispidum and G. nipponicum, were reported in Korea so far [4-7]. G. hispidum was found in the viper snake (Gloydius brevicaudus) [4], and G. nipponicum was found in the Asian tiger keelback snake (Rhabdophis tigrinus) as well as the red-tongued viper snake ( $G$. ussuriensis) $[5,6]$. Both species of Gnathostoma larvae can induce zoonosis. On the other hand, $K$. natricis detected in the intestine of Asian tiger keelback snake was described as a nonzoonotic nematode [7]. In the present study, we describe additional 3 non-zoonotic nematode species from snakes, i.e., Kalicephalus brachycephalus, Kalicephalus sinensis, and Ophidascaris excavata, recovered from Korean terrestrial snakes in the faunistic point of view.

\footnotetext{
- Received 13 November 2015, revised 7 January 2016, accepted 16 January 2016.

*Corresponding author (kseom@chungbuk.ac.kr)

(c) 2016, Korean Society for Parasitology and Tropical Medicine

This is an Open Access article distributed under the terms of the Creative Commons Attribution Non-Commercial License (http://creativecommons.org/licenses/by-nc/3.0) which permits unrestricted non-commercial use, distribution, and reproduction in any medium, provided the original work is properly cited.
}

The Chungnam Wild Animal Rescue Center (CWARC) and Korea Broadcasting System (KBS) donated the snakes to Parasite Resource Bank, Korea National Research Resource Center, the Republic of Korea (PRB) in July 2014 and February 2015, respectively. The host identification and scientific name were based on the reference, 'Ecological Guide Book of Herpetofauna in Korea' and 'National List of Species of Korea (Vertebrates)' $[8,9]$. A red-banded snake (Dinodon rufozonatm), a dione rat snake (Elaphe dione), and a viper snake (G. brevicaudus) that were collected from Nonsan-si (City) were donated by CWARC. The Programming Department of Korea Broadcasting System (KBS) donated 2 Asian tiger keelback snakes ( $R$. tigrinus) and an Amur rat snake (Elaphe schrenckii) to PRB for examination of sparganum infections. These snakes were collected from the northern region of Chungcheongbuk-do (Province). The snakes were all confiscated from illegal hunters.

The snake carcasses donated by the CWARC were preserved at $-20^{\circ} \mathrm{C}$ in a freezer until examination. The other snakes were donated alive and were euthanized with chloroform anesthesia immediately before decapitation and examination for parasites. The entire bodies of the snakes were skinned, and the skins were searched for spargana visually without magnification. The viscera were separated by organs, which were opened and washed in warm water. This washed material was filtered through $200 \mu \mathrm{m}$ sieve, and methods of sedimentation were used to recover the parasites. All parasite materials used in this 
study were provided by PRB. Parasites recovered from the hosts were stored in $70 \%$ ethyl alcohol, fixed in 10\% neutral formalin, and were transferred to $70 \%$ ethyl alcohol and glycerol in a 9:1 solution for clearing. After the alcohol dried, the specimens were mounted in glycerol jelly and observed under a light microscope. For observations of the stomal structures and the caudal papillae of the ascarid nematode (O. excavata), a scanning electron microscope (SEM) was used. The specimens were washed 3 times with PBS, fixed in $2.5 \%$ glutaraldehyde solution, and washed 3 times again with PBS. After dehydration using a graded series of ethyl alcohol (70-100\%), the nematodes were transferred twice to isoamyl acetate and then dried with a filter paper and silica gel stored in an airtight container for overnight. After gold coating, the specimens were observed under a SEM (LEO-1530l; Oberkochen, Germany).

\section{Kalicephalus brachycephalus Maplestone, 1931}

(Family Diaphanocephalidae Travassos, 1920: Genus Kalicephalus Modlin, 1861)
This nematode was recovered from the rectum of a $D$. rufozonatum and $2 R$. tigrinus. Five males and 5 females from each host were analyzed, and the morphometric data are provided in Table 1.

Host: Dinodon rufozonatum and Rhabdophis tigrinus

Habitat: Rectum of snakes

Locality: Nonsan-si (City), Chungcheongnam-do (Province) and Jecheon-si (City), Chungcheongbuk-do (Province)

General description $(n=20)$ : Body slim. Head large and compressed laterally, with a slight tilt dorsally. Oral opening dorsoventrally narrowed with 3 pairs of circumoral papillae. Each papilla led by 3 parenchymatous bands. Buccal capsule large and well developed. Anterior chitinoid ridge curved. Posterior ventral piece crescentic and elongated longitudinally. Dorsal gutter long, with ventral side beaded. Esophagus narrow, with poorly developed esophageal bulb. Nerve ring situated in the $1 / 3$ to $2 / 3$ region of the esophagus. Excretory pore situated at the level of esophagus (Fig. 1A).

Male: Body much smaller than that of females. Bursa sym-

Table 1. Measurements of Kalicephalus brachycephalus and $K$. sinensis collected from each host ( $\mu \mathrm{m})$

\begin{tabular}{|c|c|c|c|c|c|c|c|c|}
\hline $\begin{array}{l}\text { Host \& organ } \\
\text { Parasite species }\end{array}$ & \multicolumn{2}{|c|}{$\begin{array}{c}\text { Rhabdophis tigrinus Cecum } \\
\text { K. brachycephalus }\end{array}$} & \multicolumn{2}{|c|}{$\begin{array}{c}\text { Dinodon rufozonatus Cecum } \\
\text { K. brachycephalus }\end{array}$} & \multicolumn{2}{|c|}{$\begin{array}{c}\text { Elaphe dione Stomach } \\
\text { K. sinensis }\end{array}$} & \multicolumn{2}{|c|}{$\begin{array}{c}\text { Elaphe schrenckii Stomach } \\
\text { K. sinensis }\end{array}$} \\
\hline \multirow{2}{*}{ Male } & \multicolumn{2}{|c|}{$N=4$} & \multicolumn{2}{|c|}{$N=5$} & \multicolumn{2}{|c|}{$N=5$} & \multicolumn{2}{|c|}{$N=4$} \\
\hline & Variation & Average & Variation & Average & Variation & Average & Variation & Average \\
\hline Body length* & $8,903-10,986$ & $(9,796 \pm 874)$ & $8,878-9,994$ & $(9,290 \pm 493)$ & \multicolumn{2}{|c|}{$5,675-7,250(6,425 \pm 637)$} & \multicolumn{2}{|c|}{$4,638-7,192(6,014 \pm 1,049)$} \\
\hline Body width† & $372-422$ & $(398 \pm 20)$ & $372-402$ & $(388 \pm 11)$ & $250-425$ & $(345 \pm 69)$ & 285-335 & $(310 \pm 23)$ \\
\hline Head width† & $245-265$ & $(257 \pm 9)$ & $274-343$ & $(304 \pm 26)$ & $137-157$ & $(147 \pm 7)$ & $184-193$ & $(185 \pm 5)$ \\
\hline Buccal cavity depth* & $265-294$ & $(284 \pm 14)$ & $225-265$ & $(239 \pm 15)$ & $173-225$ & $(194 \pm 23)$ & $102-131$ & $(117 \pm 12)$ \\
\hline Nerve ring ${ }^{*}$ & $352-384$ & $(373 \pm 15)$ & $314-333$ & $(325 \pm 8)$ & $225-274$ & $(253 \pm 19)$ & $205-255$ & $(234 \pm 21)$ \\
\hline Excretory pore ${ }^{*}$ & $439-615$ & $(539 \pm 75)$ & $500-529$ & $(515 \pm 13)$ & $372-412$ & $(390 \pm 20)$ & $342-439$ & $(374 \pm 44)$ \\
\hline Esophagus length & $466-536$ & $(505 \pm 33)$ & $441-510$ & $(470 \pm 29)$ & $304-372$ & $(345 \pm 27)$ & $293-312$ & $(308 \pm 10)$ \\
\hline Esophagus width† & $146-198$ & $(175 \pm 22)$ & $113-186$ & $(150 \pm 34)$ & $196-225$ & $(210 \pm 11)$ & $156-186$ & $(173 \pm 15)$ \\
\hline Right spicule length & $283-293$ & $(289 \pm 4)$ & $280-298$ & $(290 \pm 6)$ & $235-280$ & $(266 \pm 18)$ & $260-293$ & $(279 \pm 14)$ \\
\hline Left spicule length & $283-300$ & $(289 \pm 8)$ & $273-298$ & $(286 \pm 10)$ & $258-283$ & $(267 \pm 11)$ & $258-298$ & $(280 \pm 17)$ \\
\hline \multirow{2}{*}{ Female } & \multicolumn{2}{|c|}{$N=5$} & \multicolumn{2}{|c|}{$N=5$} & \multicolumn{2}{|c|}{$N=5$} & \multicolumn{2}{|c|}{$N=5$} \\
\hline & Variation & Average & Variation & Average & Variation & Average & Variation & Average \\
\hline Body length & $7,539-12,350$ & $(10,494 \pm 1,858)$ & $9,722-11,358$ & $(10,640 \pm 670)$ & \multicolumn{2}{|c|}{$7,275-7,850(7,635 \pm 232)$} & $6,126-9,151$ & $(7,510 \pm 1,235)$ \\
\hline Body width $^{\dagger}$ & $372-446$ & $(409 \pm 32)$ & $400-451$ & $(440 \pm 26)$ & $400-450$ & $(435 \pm 22)$ & $372-422$ & $(405 \pm 27)$ \\
\hline Head width ${ }^{\dagger}$ & $304-323$ & $(311 \pm 9)$ & $333-353$ & $(344 \pm 11)$ & $167-176$ & $(171 \pm 5)$ & $206-248$ & $(227 \pm 19)$ \\
\hline Buccal cavity depth* & 294-304 & $(300 \pm 5)$ & $255-284$ & $(271 \pm 15)$ & $213-215$ & $(213 \pm 1)$ & $124-156$ & $(139 \pm 18)$ \\
\hline Nerve ring ${ }^{*}$ & $394-513$ & $(443 \pm 50)$ & $353-412$ & $(379 \pm 25)$ & $235-265$ & $(249 \pm 13)$ & $228-280$ & $(243 \pm 42)$ \\
\hline Excretory pore* & $531-786$ & $(605 \pm 104)$ & $441-500$ & $(488 \pm 26)$ & $275-308$ & $(295 \pm 14)$ & $234-382$ & $(329 \pm 56)$ \\
\hline Esophagus length & $570-615$ & $(587 \pm 17)$ & $500-519$ & $(514 \pm 10)$ & $333-368$ & $(349 \pm 13)$ & $312-361$ & $(352 \pm 37)$ \\
\hline Esophagus width $^{\dagger}$ & $171-221$ & $(195 \pm 24)$ & 167-196 & $(184 \pm 13)$ & $195-210$ & $(203 \pm 7)$ & $215-234$ & $(223 \pm 11)$ \\
\hline Vulva length & $4,588-8,060$ & $(6,448 \pm 1,749)$ & $5,902-6,994$ & $(6,627 \pm 470)$ & \multicolumn{2}{|c|}{$4,650-5,075(4,830 \pm 167)$} & $3,770-5,870$ & $(4,605 \pm 895)$ \\
\hline Vulva ratio & $1.49-1.88$ & $(1.64 \pm 0.21)$ & $1.55-1.93$ & $(1.71 \pm 0.17)$ & $1.48-1.83$ & $(1.73 \pm 0.15)$ & $1.60-1.89$ & $(1.74 \pm 0.13)$ \\
\hline Tail length & $231-300$ & $(268 \pm 25)$ & $314-412$ & $(367 \pm 36)$ & $285-325$ & $(301 \pm 15)$ & $234-303$ & $(279 \pm 27)$ \\
\hline
\end{tabular}

${ }^{*}$ from anterior extremity, ${ }^{\dagger}$ at the maximum width, vulva ratio: prevulva/postvulva length. 

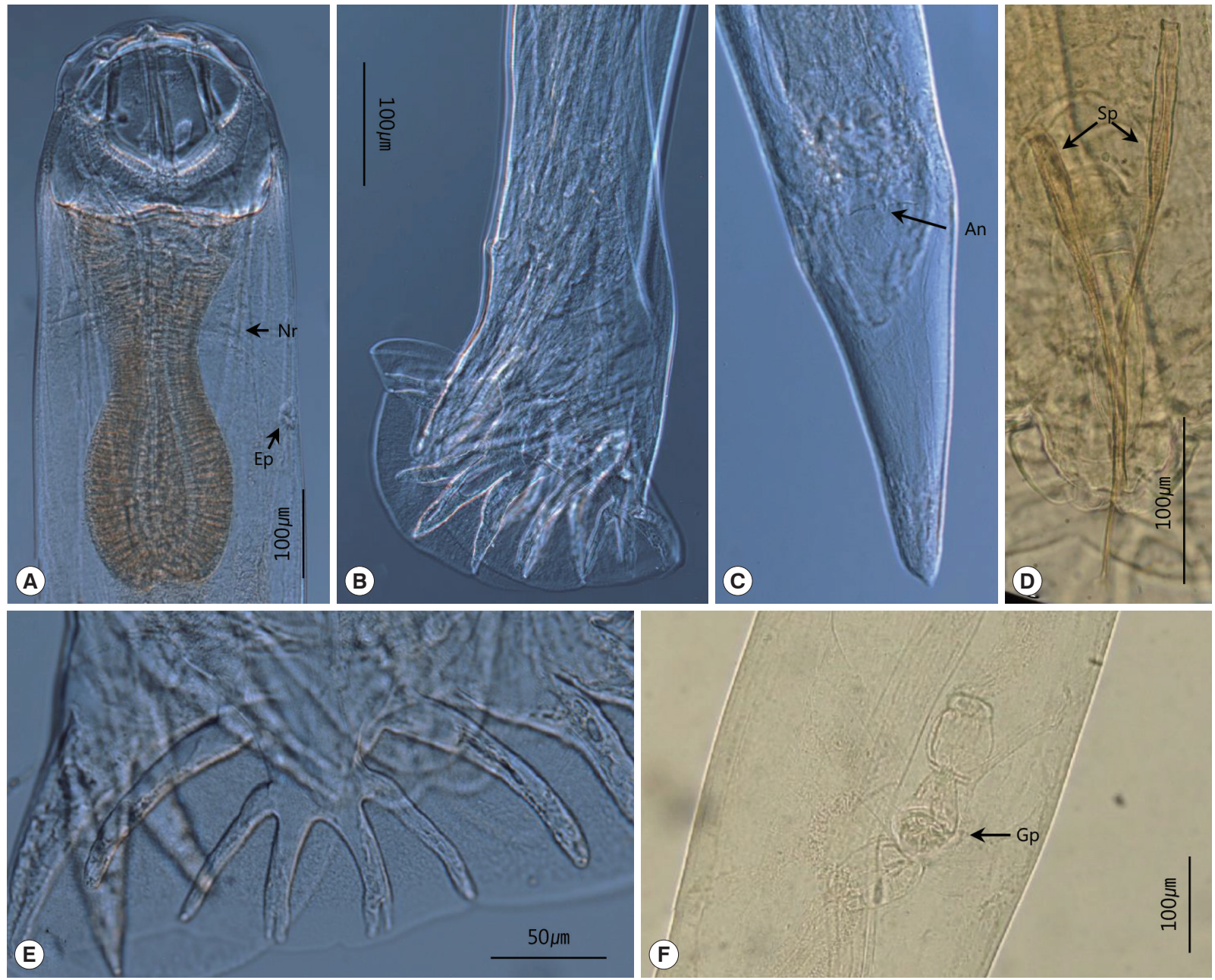

Fig. 1. Kalicephalus brachycephalus recovered from a red-sided water snake in the Republic of Korea. (A) Anterior part with the esophageal region. (B) Bursa of a male. (C) Posterior part of a female. (D) Straight spicules of a male worm. (E) Dorsal ray of the male bursa. (F) Amphidelphic disposition of the uterus. Nr, nerve ring; Ep, excretory pore; An, anus; Sp, spicule; Gp, genital pore.

metrical. Ventral ray short and situated more to the anterior than the other rays. Dorsal ray short and stout. Pairs of long and slender branches situated at the base of the dorsal ray. The main branch divided into 2 pairs of branches; middle length, outer branches and short, inner branches. The inner branches divided again at the tip at the end of the branches (Fig. 1E). Genital cone well developed, and at the tip of the cone, pairs of papillae situated (Fig. 1B). Two spicules simple, slender, and approximately similar in size, with short, spatulate tips. Gubernaculum present (Fig. 1D).

Female: Vulva located at 2/3 from anterior end, slightly projected. Amphidelphic disposition of uterus (Fig. 1F). Postvulvar region comparatively narrower than prevulvar region. Tail very short, blunted, and conical-shaped, with a small mucron (Fig. 1C).

\section{Kalicephalus sinensis Hsü, 1934}

(Family Diaphanocephalidae Travassos, 1920: Genus Kalicephalus Modlin, 1861)

This nematode was collected from the stomach of $1 E$. dione and $1 E$. schrenckii snakes. Five males and 5 females from each host were analyzed, and the morphometric data are provided in Table 1.

\section{Host: Elaphe dione and Elaphe schrenckii}

Habitat: Stomach of snakes

Locality: Nonsan-si (City), Chungcheongnam-do (Province) and Jecheon-si (City), Chungcheongbuk-do (Province).

General description: Body short, relatively plump. Head small and compressed laterally, not tilted. Posterior part of head cuticle slightly arched, with angular corners formed. Oral opening dorsoventrally narrowed with 3 pairs of circumoral 

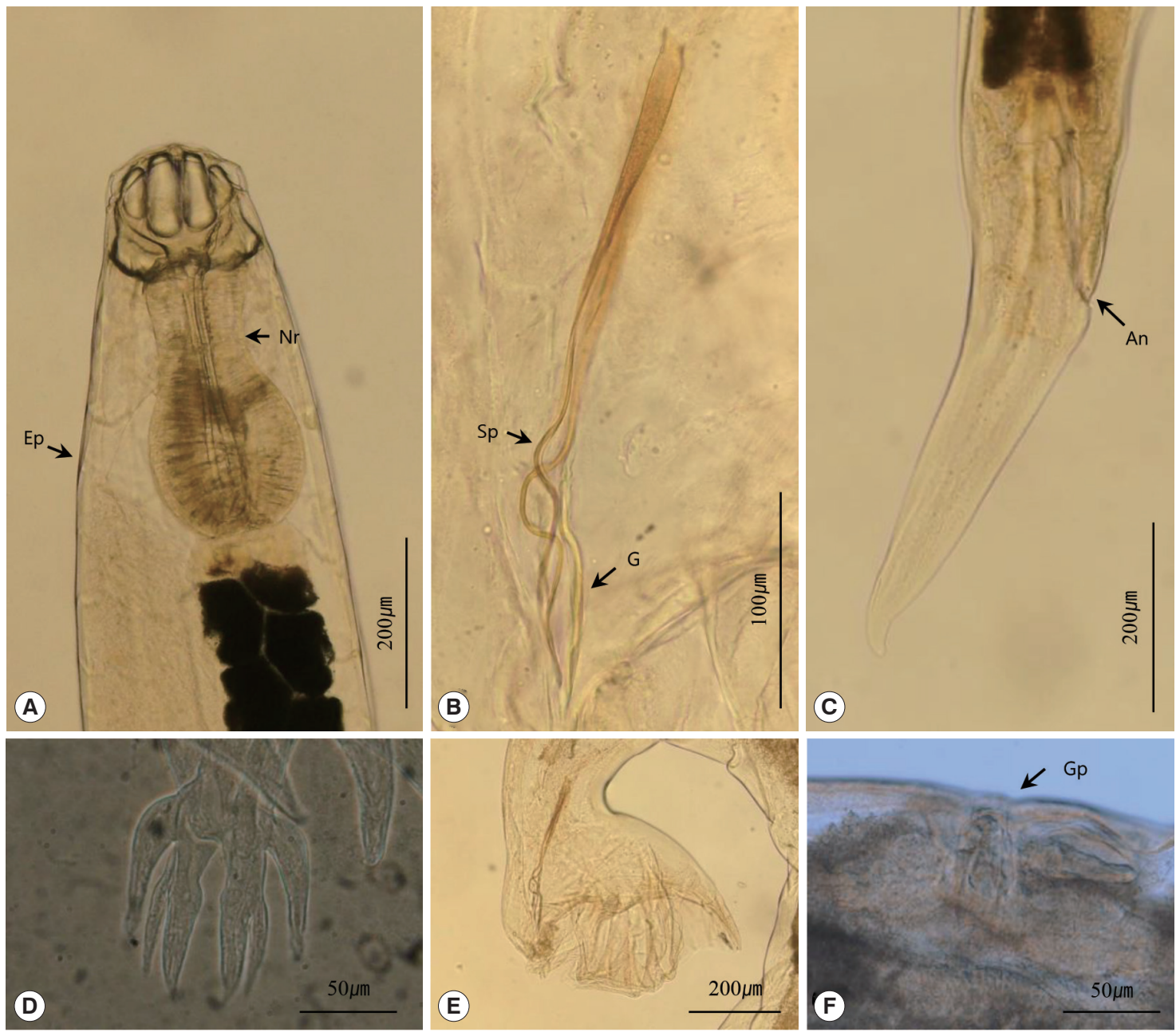

Fig. 2. Kalicephalus sinensis recovered from a rat snake in the Republic of Korea. (A) Anterior part with the esophageal region. (B) Bent spicules and gubernaculum of a male. (C) Posterior part of a female. (D) Dorsal ray of the male bursa. (E) Bursa of a male. (F) Amphidelphic disposition of the uterus. Nr, nerve ring; Ep, excretory pore; Sp, spicule; G, gubernaculum; An, anus; Gp, genital pore.

papillae. Each papillae led by 3 parenchymatous bands. Buccal capsule well developed. Face rounded. Anterior chitinoid ridge curved and narrow. Posterior dorsal and ventral pieces rounded. Dorsal gutter short and well developed. Esophagus stout, with esophageal bulb well developed. Nerve ring located at the anterior region of the esophagus. Excretory pore situated at the level of esophagus. Excretory gland large, located ventrally on the body (Fig. 2A).

Male: Body much smaller than that of females. Bursa symmetrical. Ventral ray short and situated more to the anterior than other rays. Dorsal ray short. Pairs of stout branches situated at the base of the dorsal ray (Fig. 2E). The main branch divided into 2 branches, and each branch divided again into subdivided inner branches and comparatively long outer branches (Fig. 2D). Genital cone with 2 rounded papillae on dorsal lip of cloaca. Two spicules thin, bent 1 or 2 times at each $1 / 3$ level, equal in size, with short, spatulate tips. Gubernaculum present (Fig. 2B).

Female: Vulva slightly projected but not on all of the specimens. Amphidelphic disposition of uterus (Fig. 2F). Tail short, tapered, and conical-shaped, with a pointed, small mucron (Fig. 2C).

\section{Ophidascaris excavata Hsü and Hoeppli, 1931}

(Family Ascarididae Baird, 1853: Genus Ophidascaris Baylis, 1920)

Seventeen $O$. excavata were collected from the stomach and body cavity of a G. brevicaudus, with 5 of the specimens immature. Four males and 3 females were used for morphological examinations, and 5 specimens were used for SEM. The mea- 
Table 2. Measurements of the present specimens of Ophidascaris excavata $(\mu \mathrm{m})$

\begin{tabular}{|c|c|c|c|c|}
\hline & \multicolumn{2}{|c|}{ Male $(n=4)$} & \multicolumn{2}{|c|}{ Female $(n=3)$} \\
\hline & Variation & Average & Variation & Average \\
\hline Body length* & $52,496-63,222$ & $(55,947 \pm 4,913)$ & $68,978-108,978$ & $(83,533 \pm 22,112)$ \\
\hline Body width ${ }^{\dagger}$ & $787-910$ & $(843 \pm 51)$ & 886-935 & $(918 \pm 28)$ \\
\hline Subventral lip length & $98-111$ & $(103 \pm 6)$ & $101-143$ & $(120 \pm 21)$ \\
\hline Interlabia length & $39-54$ & $(46 \pm 6)$ & $54-69$ & $(60 \pm 8)$ \\
\hline Nerve ring ${ }^{\star}$ & $421-528$ & $(475 \pm 62)$ & $441-627$ & $(539 \pm 93)$ \\
\hline Excretory pore* & $530-696$ & $(617 \pm 64)$ & $598-794$ & $(715 \pm 104)$ \\
\hline Oesophagus length & $2,631-2,903$ & $(2,792 \pm 125)$ & $2,854-3,887$ & $(3,206 \pm 590)$ \\
\hline Oesophagus $^{\dagger}$ & 271-394 & $(332 \pm 51)$ & 295-369 & $(336 \pm 38)$ \\
\hline Intestinal cecum & $477-765$ & $(598 \pm 123)$ & $627-930$ & $(753 \pm 158)$ \\
\hline Vulva* & & & $47,724-72,570$ & $(58,589 \pm 12,713)$ \\
\hline Vulva ratio & & & $1.99-3.23$ & $(2.49 \pm 0.65)$ \\
\hline Tail length & $175-216$ & $(191 \pm 19)$ & $199-212$ & $(207 \pm 7)$ \\
\hline Right spicule length & $1,254-1,431$ & $(1,267 \pm 114)$ & & \\
\hline Left spicule length & $1,186-1,509$ & $(1,325 \pm 135)$ & & \\
\hline
\end{tabular}

*from anterior extremity, ${ }^{\dagger}$ at the maximum width, vulva ratio: prevulva/postvulva length.
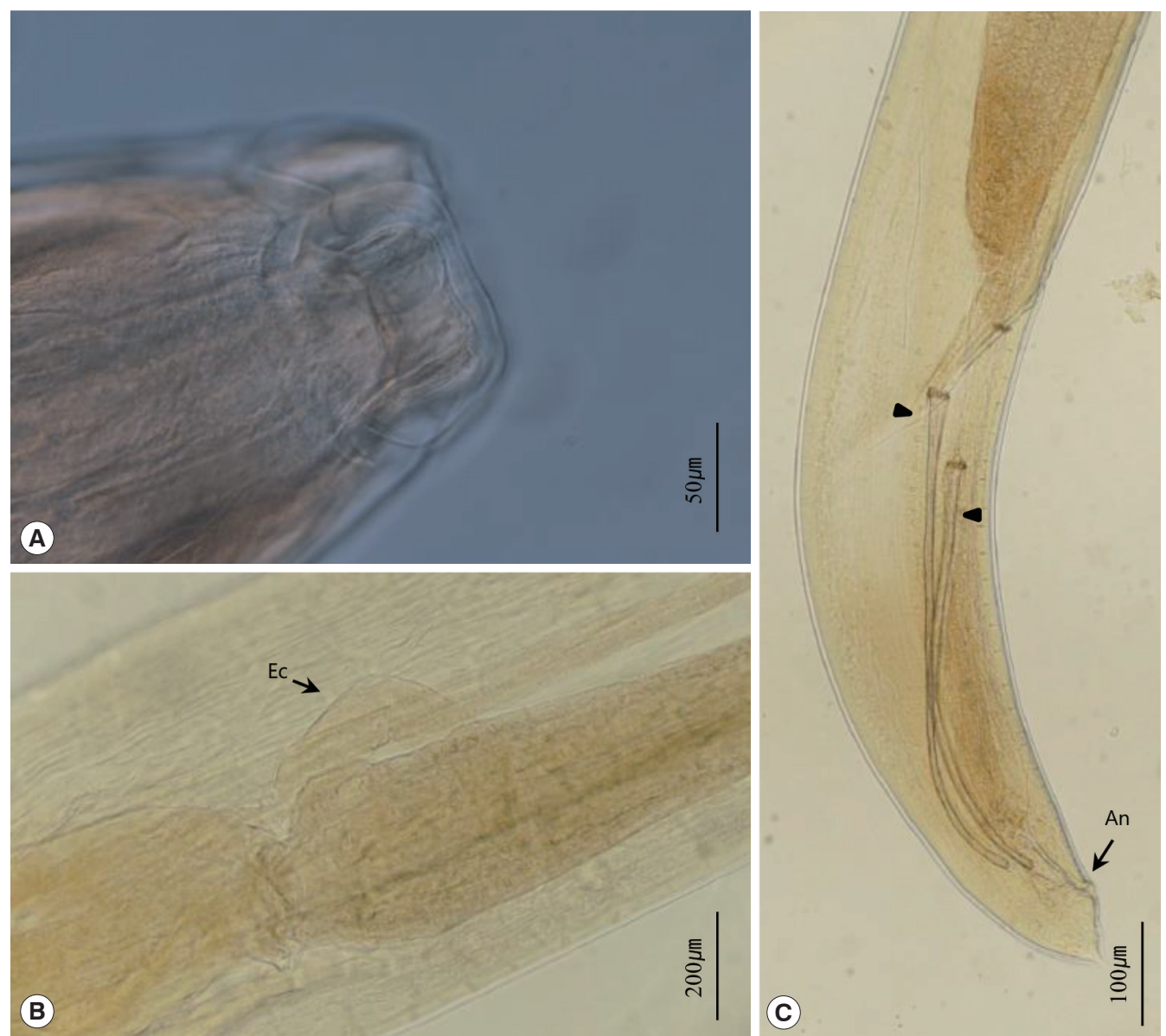

Fig. 3. Ophidascaris excavata recovered from a pit viper in the Republic of Korea. (A) Head of O. excavata. (B) Junction between the esophagus and intestine with an esophageal cecum. (C) Posterior part of a male with 2 spicules (arrow head). Ec, esophageal cecum; An, anus. 

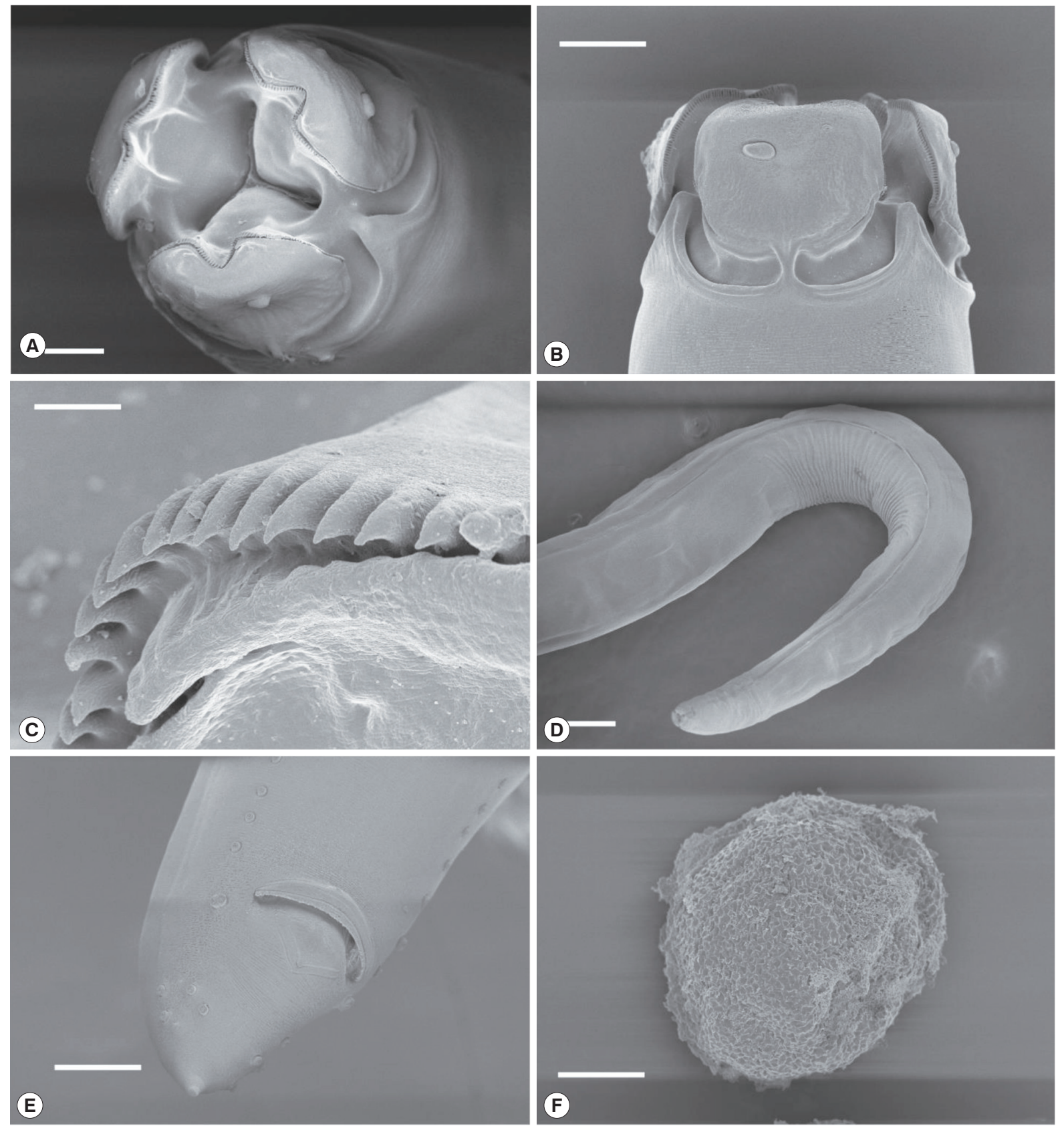

Fig. 4. Scanning electron microscopic views of Ophidascaris excavata. (A) Apical view of O. excavata (Bar=50 $\mu \mathrm{m})$. (B) Ventrolateral view of the head (Bar $=50 \mu \mathrm{m})$. (C) Dentigerous inner ridges of a lip (Bar $=10 \mu \mathrm{m})$. (D) Anterior part with narrow cervical alae $(\mathrm{Bar}=200 \mu \mathrm{m})$.

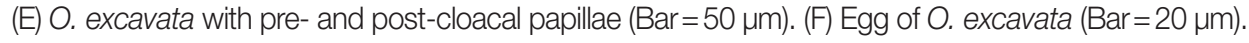

surements of these specimens are provided in Table 2.

Host: Gloydius brevicaudus

Habitat: Stomach of snakes

Locality: Nonsan-si (City), Chungcheongnam-do (Province).

General description: Body long and thin, colored bloody red immediately after necropsy. Three lips present, wider than long. Dentigerous inner ridges on each lip, with shape of ' $M$ ' or ' $W$ ' in apical view. Dorsal lip with 2 papillae, with 1 papilla each on opposite sides of the dorsal lip. Amphid located on the other side of the outer surface of each ventral lip. Interlabia well developed with deep postlabial grooves, almost half the size of the length of the lip. Isthmus narrow (Fig. 3A and Fig. 4A-C). Cervical alae narrow (Fig. 4D). Esophagus 3.6-5.0\% of 
the body length. Intestinal cecum present (Fig. 3B). Nerve ring located at the anterior part of the esophagus. Excretory pore in the region anterior to the nerve ring.

Male: Spicules thin, alate, and simple (Fig. 3C). Fifty to 58 pairs of precloacal papillae. Precloacal papillae typically single papilla but rarely double papillae. Five pairs of postcloacal papillae: 1 double, paracloacal papilla, and 2 subventral and 2 subdorsal, postcloacal papillae. Subventral and subdorsal papillae located near the posterior end. Tail short and tapered, with a mucron (Fig. 4E).

Female: Vulva located at the end of 3/4 region of the body (67-73\%). Eggs round with honeycombed surface (Fig. 4F). Tail short and rounded, with a small mucron.

The nematodes of the genus Kalicephalus are parasites of reptiles. These hookworm-like nematodes have a characteristic cephalic structure, with a laterally narrow oral opening, laterally compressed bivalval buccal cavity with complex bands, and papilla structures [10]. Two species of the genus Kalicephalus were found in this study. These nematodes were easily distinguished by the size and shape of the body, head, buccal cavity, esophageal bulb, and spicules, and the hosts and infection sites.

$K$. brachycephalus is a medium-sized kalicephalid nematode with a characteristic large buccal capsule, with a depth of over $0.27 \mathrm{~mm}$ in females and $0.20 \mathrm{~mm}$ in males, and compared with the head, relatively weak in development of the esophagus [10]. The morphological measurements of the specimens in this study were consistent with those of previous descriptions of this species [10-13]. The distribution of the nematode is in the oriental countries, including India [11], China [12], Taiwan [10], and Japan [13]. After the first record of this species from India [11], Schad [10] considered K. nankingensis Hsü, 1934, from China, as a synonym of K. brachycephalus based on the description of morphological characters. Schad [10] also examined specimens from Taiwan, which were identified as $K$. brachycephalus. This species was found previously in the Ryukyu Islands, Okinawa, Japan [13]. In the present study, we expanded the distribution of $K$. brachycephalus with a new area record in Korea.

Until this study, the species K. brachycephalus was not recorded in Korea, but Olsen [7] found 2 males and a female of the genus Kalicephalus in the intestine of a Natrix tigrina lateralis (=Rhabdophis tigrinus) from Chip'o-ri, Korea. However, Olsen [7] recorded the specimen only as K. natricis, without morphometric data and figures. Yamaguti [14] first described K. natricis in 1935 from N. tigrina and Elaphe quadrivirgata in Japan. However, Schad [10] suspected that more than 1 species was described under the name $K$. natricis, and that $K$. brachycephalus was one of the species. We support this opinion and suspect that the K. natricis reported by Olsen is a synonym of $K$. brachycephalus, particularly in consideration of the host, $R$. tigrinus.

The infection site of $K$. brachycephalus was first reported as the 'intestine' [11]. However, the parasites found from anywhere in the gut even behind the stomach are typically labeled as from the 'intestine', and therefore, the importance of distinguishing infection sites is overlooked. Schad [10] only recognized the infection sites that were identified by Hsü for $K$. brachycephalus as the rectum in 4 host species and the small intestine in 1 host species [12]. Our specimens were from the rectum of the host, which support the view of Schad [10] for the infection site.

K. sinensis is a stout nematode with short body length. The nematode has a short, well-developed esophageal bulb and 2 bent spicules [10]. K. sinensis was first described from Chinese snakes of the genus Elaphe, and other specimens have been found only from oriental snake species in the genus Elaphe [10]. Our specimens were identified from 2 Elaphe species as $K$. sinensis, based on morphological characters and the host. The hosts, E. dione and E. schrenckii, are new host records for this nematode species, and this parasite is the first record in Korea. Our specimens also resemble the descriptions of $K$. viperae (Rudolphi, 1819) Yorke and Maplestone, 1926, however, the size of the spicules was much more indicative of $K$. sinensis.

O. excavata was first described from a pit viper in Nanking, China [15]. Later, Hsü and Hoeppli [16] transferred the species from the genus Ophidascaris to the genus Amplicaecum, based on the discovery of an intestinal cecum in the species. However, Sprent [17] did not recognize the removal of this species from Ophidascaris to Amplicaecum. Sprent [17] studied the specimens in the U.S. National parasite collection from Agkistrodon halys blomhoffi, which were identified as O. excavata. The host was originally captured in Japan but died at New York Zoo. The specimens in this study were recovered from the species of pit viper (G. brevicaudus), which was originally described as the type host of $O$. excavata. The morphological characters matched well with the descriptions of O. excavata, including the intestinal cecum and the arrangement of caudal papillae. A description of the cervical alae of $O$. excavata was not included in previous studies, but in our study, we confirmed the narrow cervical alae with SEM. This is the first re- 
port of this species in Korea. Sprent [17] questioned the distribution of this parasite in Japan; however, considering the regional accessibility between Korea and Japan, this species is likely to be found in Japan.

O. shikhobalovi was originally described by Mozgovoy [18] as Amplicaecum shikhobalovi from N. natrix and Coluber ravergieri in the Moscow Zoo. After comparing it with description of Mozgovoy [18], Sprent [17] transferred its genus to Ophidascaris. However, he mentioned that it is not possible to ascertain the species as a synonym of $O$. excavata because of its longer spicule ratio to body length even though they had about the same characters with the specimens from A. $h$. blomhoffi. In $O$. schikhobalovi, the morphological characters were matched well with our specimens, including the number and arrangement of the pre- (50-52 pairs) and post-anal (6 pairs) papillae (see Mozgovoy [18]; figs. 9-17). Body lengths of young immature worms were much shorter than the adults, but size of spicules were almost similar in this study (measurements not included in this study). In our opinion, O. schikhobalovi is a synonym of O. excavata, although further comparative research is needed based on sequence analysis to make sure the status of this species.

Hsü and Hoeppli [15] noted the infection site of O. excavata was the stomach, and Sprent [17] also noted the same location. Our specimens of $O$. excavata were collected not only from the stomach but also from the body cavity. However, the host was frozen before examination, and those parasites could have escaped from their own location before completely frozen; we regard the stomach as the original site of infection.

In conclusion, we found 3 parasitic nematodes from 5 species of terrestrial snakes as new records in Korea. There exist 11 species of terrestrial snakes in Korea $[8,9]$, and many of these species have not been investigated for parasites. Therefore, further studies are required to expand Korean fauna of helminth parasites.

\section{ACKNOWLEDGMENTS}

This work was supported by a grant from the National Institute of Biological Resources (NIBR), funded by the Ministry of Environment (MOE), the Republic of Korea (NIBR no. 201402-001). Parasite materials used in this study were provided by the Parasite Resource Bank of Korea, National Research Resource Center (2012-0000037), the Republic of Korea.

\section{CONFLICT OF INTEREST}

We have no conflict of interest related to this work.

\section{REFERENCES}

1. Cho SY, Hwang KI, Seo BS. On the Sparganum mansoni infection in some Korean terrestrial snakes. Korean J Parasitol 1973; 11: 87-94.

2. Cho SY, Song KW, Lee SH. Cestode parasites of terrestrial snakes in Korea. Korean J Parasitol 1982; 7: 321-333.

3. Hong ST, Hong SJ, Lee SH, Seo BS, Chi JG. Studies on intestinal trematodes in Korea. VI. On the metacercaria and the second intermediate host of Fibricola seoulensis. Korean J Parasitol 1982; 20: 101-111.

4. Sohn WM, Lee SH. The first discovery of larval Gnathostoma hispidum (Nematoda: Gnathostomidae) from a snake host, Agkistrodon brevicaudus. Korean J Parasitol 1998; 36: 81-89.

5. Woo HC, Oh HS, Cho SH, Na BK, Sohn WM. Discovery of larval Gnathostoma nipponicum in frogs and snakes from Jeju-do (Province), Republic of Korea. Korean J Parasitol 2011; 49: 445448.

6. Han ET, Lee JH, Choi SY, Park JH, Shin EH, Chai JY. Surface ultrastructure of the advanced third-stage larvae of Gnathostoma nipponicum. J Parasitol 2003; 89: 1245-1248.

7. Olsen LS. A new species of Neoacsaris (Nematoda) from a Korean wood mouse. Trans Am Microscop Soc 1957; 76: 205-208.

8. Lee JH, Chang HJ, Seo JH. Ecological guide book of herpetofauna in Korea. National Institute of Environmental Research, Incheon, Korea. 2011, p 256.

9. Min MS, Kim JB, Shim JH, Oh HS, Park DS, Song JY. Reptile species of Korea. In National list of species of Korea (vertebrates). National Institute of Biological Resources, Incheon, Korea. 2012, pp 199-207.

10. Schad GA. Studies on the genus Kalicephalus (Nematoda: Diaphanocephalidae). II. A taxonomic revision of the genus Kalicephalus Molin, 1861. Can J Zool 1962; 40: 1035-1165.

11. Maplestone PA. Parasitic nematodes obtained from animals dying in the Calcutta zoological gardens. Rec Indian Museum 1931; 33: 71-171.

12. Hsü HF. On some Kalicephalus species from China with a discussion of certain systematic characters of the genus. Peking Nat Hist Bull 1934; 8: 375-389.

13. Hasegawa H, Otsuru M. Helminth fauna of the Ryukyu Archipelago, Japan. 2. Four Kalicephalus species parasitic to snakes in Okinawa island (Nematoda: Diaphanocephalidae). Ryukyu Univ J Health Sci Med 1982; 5: 1-10.

14. Yamaguti S. Studies on the helminth fauna of Japan. Part II. Reptilian nematodes. Jpn J Zool 1935; 6: 393-402.

15. Hsü HF, Hoeppli R. Parasitic nematodes mostly from snakes collected in China. Chinese Med J 1931; 17: 567-588.

16. Hsü HF, Hoeppli R. Miscellaneous observations on ten species 
of parasitic nematodes. Chinese Med J 1938; Suppl No. 2: 451460.

17. Sprent JFA. Ascaridoid nematodes of amphibians and reptiles:
Ophidascaris Baylis, 1920. Syst Parasitol 1988; 11: 165-213.

18. Mozgovoy AA. On the anisakid fauna of fishes and reptiles. Trudy Gel'mint Lab 1950; 3: 102-118. 